\title{
Linguistic focus and memory: An eye movement study
}

\author{
Peter Ward and Patrick Sturt \\ University of Glasgow, Glasgow, Scotland
}

\begin{abstract}
We report an eyetracking study investigating the effects of linguistic focus on eye movements and memory during two readings of a text. Across two presentations of the text, a critical word either changed to a semantically related word or remained unchanged. Focus on the critical word was manipulated using context. Eye movements were monitored during reading, and there was a secondary task of detecting the word change. Results indicated that when a word changed, participants were more successful at detecting it when it was in focus. In the second display, there were more fixations and longer viewing times on a changed than on anchanged word, but only when the critical word was in focus; eye movement data for changed and unchanged words did not differ when the word was not in focus. We suggest that linguistic focus leads to more detailed lexical semantic representations but not more effortful initial encoding of information.
\end{abstract}

The focus of a sentence has been defined as the information that is most prominent or emphasized within it (Chomsky, 1971; Halliday, 1967). There are a number of ways in which linguistic entities can become focused. In written language, information about focus can be conveyed through syntactic structure or context, but in spoken language, focus can also be conveyed by intonation. The term focus has been used in many different ways in the literature: In the discourse processing literature, for example, focus often refers to a salient individual in the discourse, such as the first character to be mentioned (Gernsbacher \& Hargreaves, 1988). However, in this article we use the term focus in a slightly different, semantic sense. For example, in the default interpretation of the cleft sentence below, the cleft element John is in focus:

(1) It was John who married my sister.

In terms of semantics, the sentence implies that John is being singled out as the person who married the sister, in contrast to other individuals who might have done so (Rooth, 1995). Note that a sentence that includes a focused entity can be naturally interpreted as an answer to an implied question. In Example 1, the sentence is a natural answer to the question Who married your sister? The idea of focus supplying the answer to some implied question becomes particularly clear when we consider the use of embedded questions as a focusing device, as in Example 2:

(2) Susan asked her son which prize he had won.

It was the prize for spelling.

In this case, the focusing question introduced by which prize is embedded in the first sentence, resulting in an interpretation of the second sentence in which focus falls on the phrase the prize for spelling.

There are a number of processing benefits associated with focused items. Listeners detect target phonemes more quickly when they are part of focused phrases in a phoneme monitoring task (Cutler \& Fodor, 1979). Moreover, readers are more likely to detect an anomaly when it occurs as part of the sentence focus. For example, under normal conditions, people often fail to notice the anomaly in the Moses illusion (Erickson \& Mattson, 1981): They typically do not notice that Noah rather than Moses is the one who should be named in the sentence Moses put two of each kind of animal onto the ark. However, Bredart and Modolo (1988) showed that people are much more likely to notice the anomaly when Moses is focused by means of a cleft construction, as in It was Moses who put two of each kind of animal onto the ark. Moreover, Gergely (1992) found that focusing relevant information facilitates inference, and Birch and Garnsey's (1995) experiments on focus and memory demonstrated a strong impact of linguistic focus on the memory representations for sentences, showing that target words identical to previously focused words were facilitated in a recognition task both with and without a delay.

Sturt, Sanford, Stewart, and Dawydiak (2004) used a new technique to further investigate the effects of focus on memory. Their text change detection paradigm drew from the literatures on change blindness in visual scene viewing (Rensink, O'Regan, \& Clark, 1997) and on the rereading of previously read text (Raney \& Rayner, 1995). In Sturt et al.'s study, participants read two successive presentations of a short text passage with an interposing blank screen, and a single word was changed between presentations. This technique is similar to that used by Raney and Rayner in their study of repetition effects across two 
readings of a text. Raney and Rayner's eye movement data revealed an overall processing facilitation on the second reading, with readers in general making fewer and shorter fixations, as well as longer saccades. Interestingly, their experimental passages contained a target word that changed to a synonym in the second presentation in some conditions, and although detection of word replacement was not tested online and a word was changed in only half of the trials, the participants reported being unaware that substitutions had been made. See Raney (2003) for an extensive overview of repetition effects in rereading.

The Sturt et al. (2004) study differed from Raney and Rayner (1995), however, in that the participants were required to identify the word change, if any, and that eye movements were not monitored. In the experimental trials, the critical word either changed to a semantically related word between the first and second text displays (e.g., hat changed to cap) or changed to a semantically unrelated word (e.g., hat changed to $d o g$ ). Furthermore, the critical word was embedded in a noun phrase (NP) that either was or was not focused, with focus manipulated via clefting (Experiment 1) or embedded questions in the context (Experiment 2). An example item from the focus condition of Experiment 2 is given below, with the critical word in bold:

(3) Everybody was wondering which man got into trouble.

In fact, the man with the hat was arrested.

The nonfocus conditions were similar, except that the embedded question in the initial sentence did not result in the critical NP becoming focused (e.g., Everybody was wondering what was going on that night).

Sturt et al. (2004) reported an effect of focus on change detection performance: When the critical word changed to a semantically related word, detection rates were higher for words that were in focus, but detection rates were unaffected by focus when the critical word changed to a semantically unrelated word. Sturt et al. interpreted their results in terms of the representation of a word's meaning, arguing that the level of granularity at which a word is represented could be modulated by factors such as focus (see Hobbs, 1985, for detailed discussion of the issue of granularity). For example, a word like cap might be represented more generally as headgear, or more specifically as a flat piece of headgear with a sun shade. Sturt et al. claimed that focus increases the specificity with which a word is represented. Thus, a change from hat to cap was noticed relatively often in the focus condition, because the relevant semantic information had been represented at a finer-grained level. In contrast, when the phrase was not in focus, a less specific representation of the meaning led to a relatively lower level of change detection. However, with more distant semantic changes, in which a word changed to a word in a completely different semantic category (e.g., hat changing to dog), the change was typically detected successfully, irrespective of focus.

This phenomenon of variable specificity in the representation of word meaning is consistent with recent claims that representations are built at a level of detail that is "good enough" for the relevant task (see Ferreira, Bailey, \& Ferraro, 2002; Sanford \& Sturt, 2002).
However, on the basis of the detection data reported by Sturt et al. (2004), it is not possible to make conclusions about the underlying processes that result in the focus benefit that they observed. In fact, several hypotheses could explain the result. One possibility, suggested by Birch and Rayner (1997), is that enhanced memory representations for focused information "may be due in part to differences in reading patterns for focused information" (Birch \& Rayner, 1997, p. 653). In their eyetracking studies, Birch and Rayner found that readers spent longer rereading focused words than nonfocused words. The researchers also found that initial (first-pass) reading times were longer for multiword phrases when those phrases were in focus. Birch and Rayner claimed that the higher number of fixations and longer processing times led to an encoding benefit for the focused information. In terms of the Sturt et al. results, if such differences in reading behavior occurred when the participants read the first text display, this could partially explain their enhanced performance in detecting changes to focused words in the second display. It is true that, on some trials, rather than fixating a focused critical word more often, readers might not have fixated it at all during the first display. (Although readers may process a word parafoveally in many trials, in other trials they may not process the word at all when it is not fixated.) But if readers did make more fixations on focused phrases in the first display, at least some component of the focus benefit on subsequent change detection could be explained in strictly oculomotor terms.

However, the claim that readers fixate longer on focused words is not universally supported by the evidence. In fact, Morris and Folk (1998) found that total fixation times were actually shorter for focused than for nonfocused words, and they claimed instead that focused information was easier to integrate into the discourse representation than nonfocused information. Enhanced integration of focused information might lead to more detailed memory representations, which also could explain the performance benefit for focused items in Sturt et al.'s (2004) change detection task.

In summary, although there has been evidence that focus enhances both memory representations (Birch \& Garnsey, 1995) and the level of detail with which word meanings are represented (Sturt et al., 2004), it is currently unclear how these benefits relate to online reading behavior. In the present study, we used Sturt et al.'s text change detection task, but we also recorded eye movements during reading. The offline accuracy results for the change detection task would allow an opportunity to replicate the effect of focus reported by Sturt et al., and the eye movement record should allow inferences to be drawn about the processing effects of focus. Analysis of reading behavior in the first display would indicate the extent to which any focus benefits were due to differences in the number and duration of fixations in the encoding phase. Analysis of such behavior in the second display would indicate how and when any effects of focus manifested themselves in processing. Specifically, if focus leads to more detailed representations of lexical semantic information, we would expect a greater slowdown on a changed word when that word was in focus than when it was not. 
In addition to these benefits, the eye movement data allowed us the possibility of finding implicit effects of stimulus changes in the eye movement record. The literature on change detection in visual cognition has shown evidence for increased fixation times on changed elements, even in trials in which participants failed to detect the change (see, e.g., Hollingworth \& Henderson, 2003). In other words, the cognitive system sometimes registers the fact that a change occurs without this information reaching the level of conscious awareness. The present experiment provided the opportunity to examine whether such behavior by the cognitive system generalizes to linguistic stimuli.

\section{METHOD}

\section{Participants}

Thirty-two participants were recruited from the University of Glasgow student community. All participants were native English speakers, had not been diagnosed with dyslexia, and had either normal vision or corrected-to-normal vision using soft contact lenses. All participants were naive to the design and goals of the study.

\section{Materials and Design}

In the experimental design, we manipulated two factors: whether or not the critical word changed (change vs. no change) and whether or not the critical word was in focus (focus vs. nonfocus). We created 24 experimental items like those in Example 4 below, in two focus conditions (see the Appendix; the notation Word $1 \rightarrow$ Word 2 indicates that, in the change conditions, Word 1 changes to Word 2 between the two displays; in the no-change conditions, Word 2 was used as the critical word in both displays).

(4) A. Focus

I couldn't decide which seat to take at the theatre. I hoped the seat by the exit $\rightarrow$ door would give me a good view.

It turned out to be a wonderful evening's entertainment.

B. Nonfocus

I couldn't decide whether I liked the new theatre layout.

I hoped the seat by the exit $\rightarrow$ door would give me a good view.

It turned out to be a wonderful evening's entertainment.

In the focus conditions (4A), the critical word was focused by manipulating the structure of the opening sentence to include context focus, or an embedded question. In the nonfocus conditions (4B), the opening sentence did not include a structure that placed focus on the critical word. In the focus conditions, the opening sentence always contained an embedded which question followed by an NP (e.g., which seat ...). In the second sentence, the head noun was repeated and followed by a modifier (e.g., the seat by the door). The critical word always occurred within the modifier, as in Sturt et al. (2004). We did not use the head noun of the critical NP (e.g., seat) as the critical word because this word would be repeated from the first sentence in the focus conditions but not in the nonfocus conditions. Thus, it was thought that using another noun within the modifier (e.g., door) would allow us to examine effects of focus without the possibility of any processing benefits due to repetition between the first and second sentences.

Note also that the critical word above, door, provides important semantic information because it serves to single out one particular seat from a hypothetical set of alternatives (i.e., the seat by the door is contrasted with seats in other locations). It is in this sense that we refer to the conditions containing embedded questions as the focus conditions.

In the nonfocus conditions, the which $N P$ construction in the opening sentence was replaced with a different wh question that did not focus on the critical word (e.g., I couldn't decide whether I liked the new theatre layout).

In the change conditions, the critical word changed to a semantically similar word between the first and second presentations of the text. Note that the levels of the change variable differ from those in Sturt et al.'s (2004) study, in which all experimental conditions included a change to a word that was either close or distant semantically. Our inclusion of baseline no-change conditions allowed us to examine what effect, if any, a changed word had on the eye movement record, over and above the expected effects for repeated words. Although all items were designed to include a close semantic change in the change conditions, some of the word changes could be regarded as involving synonyms (e.g., rucksack/backpack), and others were semantically close substitutions (e.g., kitten/puppy). According to the WordNet lexical database (developed at the Cognitive Science Laboratory at Princeton University), four of our items involved synonyms, and these are marked with an asterisk in the Appendix. ${ }^{1}$ Across conditions, the critical word was always identical in the second display-only the critical word in the first display differed between change conditions. We did this to ensure that reading times on the critical region in the second display were always measured for the same word, thus avoiding any confounds due to lexical differences. Note that in the first display, change is a dummy factor, and we are interested only in the effects of focus in this display.

Words and their changed variants were matched as closely as possible on length and frequency in order to rule out any effects caused by differences in the length or frequency of the critical word across displays. Mean critical word length was 6.67 characters in the first display and 6.75 characters in the second display. Word length ranged from 4-9 characters in the first display and from 4-10 characters in the second display. Frequency information was extracted from the 90-million-word written section of the British National Corpus (1995), and raw frequencies underwent log transformation to enable the use of a parametric means comparison. The mean $\left(\log _{10}\right)$ frequency for the critical word was 3.04 in the first display and 3.16 in the second display. Log frequencies per 90 million words ranged from 2.29 to 4.26 in the first display and from 1.32 to 4.38 in the second display. A paired-samples $t$ test indicated that neither mean word length nor mean log frequency differed significantly across displays (all $t \mathrm{~s}<1.01, p \mathrm{~s}>.1$ ).

As well as balancing length and frequency, we ensured that the two relevant critical words in the change conditions were chosen to be equally plausible within the given context. A separate norming study $(N=20)$ was carried out to ensure an equally plausible fit with context. In this study, the participants read passages from Display 1 and Display 2 in the focus/change condition. The items were divided into two lists, so that each participant saw each item in only one of its two display conditions. Participants rated each passage for plausibility on a scale from 1 to 7 points, yielding mean plausibility ratings of 5.61 for Display 1 and 5.69 for Display 2. Withinparticipants and within-items ANOVAs indicated that the difference between means was not significant (both $t \mathrm{~s}<1$ ).

Each experimental item consisted of three sentences, and the critical region was always in the second sentence. Each sentence was presented on a separate line, with no line exceeding 75 characters (including spaces) in length. The 24 experimental items were embedded among 32 filler items to prevent participants from identifying the experimental items and employing nonnatural reading strategies. Half of the filler items included changes. Thus, each participant read a total of 56 passages, displayed twice, exactly half of which included changes.

All manipulations were made within participants using a Latin square design. In this design, each participant saw each item in only one of its four focus/change conditions, but each item was seen in all four conditions across the four participant groups. 


\section{Apparatus}

Eye movements were monitored using a Generation 5.5 Fourward Technologies Dual-Purkinje-Image Eyetracker. The tracker monitored a participant's gaze location every millisecond, and the software sampled the tracker's output to establish the position of eye fixations and their start and finish times. The tracker only monitored movements of the right eye, though viewing was binocular. The text passages were displayed on a PC VDU screen positioned approximately $80 \mathrm{~cm}$ from the participants' eyes. The screen displayed approximately four characters per degree of visual angle. Participants' head movements were minimized using a bitebar (prepared individually for each participant), forehead rests, and a head strap.

\section{Procedure}

The participants were instructed that they would be reading short passages that would be presented twice, and that their task was to indicate whether a word change had occurred between the first and second presentations. They were instructed to read at their normal pace and to read through each of the two displays of any given passage only once. It was hoped that this would encourage readers to read each display in the most natural way, without continually rereading a passage from the beginning, either to memorize the first display of the text or to locate any change in the second display (Raney \& Rayner, 1995, gave their subjects similar instructions). Nevertheless, we did expect to see regressions in the eye movement data, simply because they are a normal and automatic part of the reading process. Two of our reading time measures, total reading time and number of fixations, take such rereading into account and will be included in our analysis.

Participants were not advised how often a word change was likely to occur or where in the passage a change might occur. Prior to the experiment proper, each participant completed three practice trials and a brief calibration procedure was carried out.

The experiment began with a participant fixating a small, boxshaped character in the top left section of the monitor that signaled the position of the first character of the upcoming text. The first display of the text was then presented, and the participant read through it. The participant then fixated another box-shaped character below and to the right of the last character of text and pressed either of two handheld buttons. The screen went blank, except for a capital $X$ character that appeared in the position of the first character of the text; this screen was presented for $500 \mathrm{msec}$ before being replaced by the second text display. Participants read through the second display of the passage, fixated on the passage-end box, and pressed either of the two buttons. The question screen was then displayed:

\section{No change $<>$ Change}

Participants responded by pressing the right button if they thought there had been a change or the left button if they thought there had not been a change. This sequence of screens constituted 1 trial, and the pattern was repeated throughout all 56 trials. Calibration was checked between trials, and the eyetracker was recalibrated if necessary.

\section{Data Analysis}

An automatic procedure pooled short contiguous fixations. This procedure merged fixations of less than $80 \mathrm{msec}$ into any neighboring fixations within a distance of one character and then deleted any remaining fixations of less than $80 \mathrm{msec}$.

In order to calculate eye movement measures, the experimental materials were split into regions. The regions for the item in Example $4 \mathrm{~B}$ are given below.

$$
\begin{aligned}
& {\left[{ }_{1}\right. \text { I couldn't decide whether I liked the new theatre }} \\
& \text { layout. } \\
& \text { I hoped the }]\left[{ }_{2} \text { seat }\right]\left[{ }_{3} \text { by the }\right]\left[{ }_{4} \text { door }\right]\left[{ }_{5}\right. \text { would give }
\end{aligned}
$$$$
\text { me a good view]. }
$$

For each item, the first region comprised all words up to, but not including, the head noun (e.g., seat) of the focused phrase. This region contained the embedded question in the focus conditions. The second region consisted of the head noun. The third region (e.g., by the) consisted of the words between, but not including, the head noun and the critical noun. The fourth region consisted of the critical noun (e.g., door), and the fifth region consisted of the remainder of the critical sentence.

We will report results for Regions 2-5, focusing mainly on the critical word (Region 4). We calculated standard eye movement measures associated with both early and later processing of the critical region:

The duration of the first fixation in a region is a measure of the very earliest processing in that region and is calculated by taking the duration of the fixation following the first saccade into the region from the left, before any material to the right of the region has been fixated. We also report gaze duration. This measure involves summing the duration of all fixations made within a region from the time it is first entered from the left to when it is first exited, either to the left or the right. Thus, gaze durations tend to be longer than firstfixation times, since they allow for multiple initial fixations within a region. Gaze duration is also informative about early processing, but when applied to a postcritical region, it can also be informative about later, integrative processing. The term gaze duration is generally preferred when the region of interest consists of a single word; however, this measure is generally known as the first-pass reading time when the region consists of two or more words. Since we will be reporting data for both single-word and larger regions, we will use both terms to refer to this measure.

Total time is the sum of the durations of all fixations made within a region, so it includes gaze duration/first-pass times as well as the sum of any fixations made within the region after the reader has already exited it. Like total time, the total number of fixations in a region is a more global measure of processing difficulty and is indicative of the integration of a word with its preceding context or the rereading of a word as a result of later processing disruption.

The calculation of reading time measures involved the exclusion of zero reading times from the means (i.e., trials in which a region was not fixated by the reader do not contribute to the relevant mean). In the present experiment, excluding these trials resulted in losses of $11.6 \%$ of the first-display data and $16.1 \%$ of the second-display data, averaged across regions. This did not result in any missing design cells in our critical regions, and the results for Display 1 (corresponding to a per-region average probability of .88 for first-pass fixation) are typical for reading studies in our laboratory.

\section{RESULTS AND DISCUSSION}

The eye movement measures were computed on the data for both the first and the second displays. To summarize the results, focus had no effect on reading behavior on the critical word in the first display, but reading behavior on the critical word in the second display was affected by both the focus and change manipulations. There was also some evidence that in the first display, reading proceeded more quickly in the two regions preceding the critical word in the focus conditions.

\section{First-Display Data: Did Focus Affect Initial Fixation Behavior?}

We will look first at the reading time data from the first display of the experimental passages, comparing fixation data between the focus and nonfocus conditions. As discussed in the introduction, if focus effects on the critical word in the second display are to be explained in terms of reading behavior on the first display, we would expect to find differences in reading behavior on the critical word in this display. 
For each reading time measure, two ANOVAs were computed, one by participants $\left(F_{1}\right)$ and one by materials (or items; $F_{2}$ ). The analyses for the first display were collapsed over the change variable.

The means for Regions 2-5 are presented in Table 1, where reading times are given in milliseconds.

In Region 2, there were no significant effects with firstfixation time (both $F \mathrm{~s}<1.5$, both $p \mathrm{~s}>.2$ ). For gaze duration, there was a main effect of focus, significant in the participants analysis, such that fixation durations were longer for the nonfocused items in comparison with the focused items $\left[F_{1}(1,32)=6.15, M S_{\mathrm{e}}=2,262, p<.05 ; F_{2}(1,23)=\right.$ $\left.3.18, M S_{\mathrm{e}}=2,197, p=.088\right]$. This effect was also present in the number of fixations $\left[F_{1}(1,31)=8.83, M S_{\mathrm{e}}=0.057\right.$, $\left.p<.01 ; F_{2}(1,23)=7.62, M S_{\mathrm{e}}=0.051, p<.05\right]$. In total time, this effect was marginal in the participants analysis and nonsignificant in the items analysis $\left[F_{1}(1,31)=3.74\right.$, $M S_{\mathrm{e}}=2,905, p=.062 ; F_{2}(1,23)=2.21 . M S_{\mathrm{e}}=2,130$, $p=.15]$. This advantage for the focus conditions could be attributed to repetition effects, as discussed below.

In Region 3, the precritical region, there was some evidence for a main effect of focus in the participants analysis of total time $\left[F_{1}(1,31)=6.43, M S_{\mathrm{e}}=3,467\right.$, $\left.p<.05 ; F_{2}(1,23)=3.87, M S_{\mathrm{e}}=3,417, p=.061\right]$ and in number of fixations $\left[F_{1}(1,31)=4.73, M S_{\mathrm{e}}=0.14, p<\right.$ $\left..05 ; F_{2}(1,23)=2.24, M S_{\mathrm{e}}=0.20, p=.15\right]$. Again, the tendency was for the focus conditions to be read more quickly and with fewer fixations. The effect did not reach significance in any other measure (all other $p$ s $>.05$ ).

In Region 4, the critical region, the effect of focus did not reach significance in any measure (all $F \mathrm{~s}<1$ ).

In Region 5, the only analysis in which the focus effect approached significance was for the number of fixations, in which there was marginal effect in the analysis by items only $\left[F_{1}(1,31)=2.27, p=.14 ; F_{2}(1,23)=3.51, M S_{\mathrm{e}}=\right.$ $0.030, p=.074]$. The tendency was as before, with a trend toward fewer fixations in the focus condition. The focus effect did not approach significance in any other measure (all $F_{\mathrm{s}}<1$ ).

To summarize the results for the first display, all significant effects of focus involved regions preceding the critical word. The general finding was that reading proceeded more quickly for the focus conditions than for their nonfocus counterparts in the regions before the critical word. This effect could be related to the fact that the head noun (e.g., the seat near the door) was repeated from the first sentence in the focus conditions, or it could indicate that the focus condition led to more successful integration of the content of the second sentence. Of course, these ideas are not necessarily independent of each other, since repetition itself could lead to easier integration.

The important finding, however, is that there were no detectable differences on the critical word itself. This means that any effects of focus on the critical word in the second display data cannot be explained in terms of fixation behavior in the first display.

\section{Second-Display Data}

We look next at the results from the second display of the passage, the display in which the word change occurred in the change conditions. Analysis of this display allows us to examine whether a changed critical word affects fixation behavior on that word and whether focus modulates this effect. The second display was analyzed as a function of both change and focus. When the two factors interacted significantly, we ran planned comparisons in order to compare the change and no-change conditions at each level of the focus manipulation. All means for Regions 2-5 in Display 2 (as well as for an extended critical region; see below) are presented in Table 2 .

Statistical analysis revealed no significant effects in the second display before the critical word. Below, we give the analysis results for the critical word itself.

Fixations on the critical word. Analysis of firstfixation times and gaze durations revealed a main effect of change, such that initial fixations were longer when the word changed than when it did not [for first fixation, $F_{1}(1,31)=9.46, M S_{\mathrm{e}}=1,913, p<.01 ; F_{2}(1,23)=10.15$, $M S_{\mathrm{e}}=1,508, p<.01$; for gaze duration, $F_{1}(1,31)=9.77$, $M S_{\mathrm{e}}=3,825, p<.01 ; F_{2}(1,23)=8.60, M S_{\mathrm{e}}=2,840, p<$ $.01]$. No other effects approached significance for either first fixation or gaze duration (all $F \mathrm{~s}<1.70$, all $p \mathrm{~s}>.2$ ).

Table 1

Means for the First Display, Collapsed Over the Change Variable

\begin{tabular}{|c|c|c|c|c|c|c|c|c|}
\hline \multirow[b]{2}{*}{ Measure } & \multicolumn{2}{|c|}{$\begin{array}{c}\text { Region } 2 \\
\text { seat }\end{array}$} & \multicolumn{2}{|c|}{$\begin{array}{l}\text { Region } 3 \\
\text { by the }\end{array}$} & \multicolumn{2}{|c|}{$\begin{array}{c}\text { Region } 4 \\
\text { (Critical) } \\
\text { door }\end{array}$} & \multicolumn{2}{|c|}{$\begin{array}{c}\text { Region } 5 \\
\text { would ... view }\end{array}$} \\
\hline & $M$ & $S D$ & $M$ & $S D$ & $M$ & $S D$ & $M$ & $S D$ \\
\hline \multicolumn{9}{|l|}{ First Fixation (msec) } \\
\hline Focus & 266 & 53 & 260 & 39 & 270 & 45 & 262 & 41 \\
\hline Nonfocus & 275 & 50 & 261 & 33 & 271 & 46 & 257 & 41 \\
\hline \multicolumn{9}{|c|}{ Gaze Duration/First-Pass Time (msec) } \\
\hline Focus & 282 & 63 & 373 & 73 & 305 & 59 & 836 & 195 \\
\hline Nonfocus & 312 & 75 & 390 & 100 & 312 & 68 & 854 & 205 \\
\hline \multicolumn{9}{|l|}{ Total Time (msec) } \\
\hline Focus & 320 & 78 & 453 & 96 & 362 & 90 & 967 & 262 \\
\hline Nonfocus & 346 & 68 & 491 & 128 & 353 & 77 & 987 & 225 \\
\hline \multicolumn{9}{|l|}{ Number of Fixations } \\
\hline Focus & 0.90 & 0.27 & 1.71 & 0.40 & 1.22 & 0.30 & 3.79 & 1.01 \\
\hline Nonfocus & 1.03 & 0.25 & 1.86 & 0.60 & 1.21 & 0.27 & 4.01 & 0.92 \\
\hline
\end{tabular}


Table 2

Means for Regions 2-5 and the Extended Region in Display 2

\begin{tabular}{|c|c|c|c|c|c|c|c|c|c|c|}
\hline \multirow[b]{2}{*}{ Measure } & \multicolumn{2}{|c|}{$\begin{array}{c}\text { Region } 2 \\
\text { seat }\end{array}$} & \multicolumn{2}{|c|}{$\begin{array}{l}\text { Region } 3 \\
\text { by the }\end{array}$} & \multicolumn{2}{|c|}{$\begin{array}{l}\text { Region } 4 \\
\text { door }\end{array}$} & \multicolumn{2}{|c|}{$\begin{array}{c}\text { Region } 5 \\
\text { would ... view }\end{array}$} & \multicolumn{2}{|c|}{$\begin{array}{l}\text { Ext. Region } \\
\text { by the door }\end{array}$} \\
\hline & $M$ & $S D$ & $M$ & $S D$ & $M$ & $S D$ & $M$ & $S D$ & $M$ & $S D$ \\
\hline \multicolumn{11}{|l|}{ First Fixation (msec) } \\
\hline Focus/no change & 256 & 40 & 241 & 47 & 260 & 39 & 255 & 39 & - & \\
\hline Focus/change & 252 & 74 & 245 & 52 & 288 & 86 & 262 & 44 & - & \\
\hline Nonfocus/no change & 264 & 62 & 246 & 59 & 258 & 36 & 256 & 35 & - & \\
\hline Nonfocus/change & 244 & 67 & 251 & 64 & 277 & 63 & 266 & 47 & - & \\
\hline \multicolumn{11}{|c|}{ Gaze Duration/First-Pass Time (msec) } \\
\hline Focus/no change & 270 & 50 & 315 & 83 & 280 & 54 & 681 & 196 & 460 & 115 \\
\hline Focus/change & 270 & 90 & 326 & 95 & 330 & 129 & 740 & 238 & 545 & 171 \\
\hline Nonfocus/no change & 280 & 76 & 325 & 89 & 286 & 61 & 670 & 218 & 487 & 152 \\
\hline Nonfocus/change & 264 & 72 & 316 & 88 & 304 & 75 & 724 & 257 & 483 & 120 \\
\hline \multicolumn{11}{|l|}{ Total Time (msec) } \\
\hline Focus/no change & 289 & 80 & 370 & 91 & 322 & 88 & 816 & 218 & 586 & 133 \\
\hline Focus/change & 302 & 102 & 386 & 104 & 388 & 122 & 838 & 213 & 677 & 176 \\
\hline Nonfocus/no change & 283 & 65 & 379 & 106 & 331 & 78 & 793 & 220 & 598 & 159 \\
\hline Nonfocus/change & 279 & 63 & 354 & 102 & 347 & 87 & 832 & 233 & 597 & 146 \\
\hline \multicolumn{11}{|l|}{ Number of Fixations } \\
\hline Focus/no change & 0.80 & 0.26 & 1.39 & 0.44 & 1.00 & 0.30 & 3.26 & 0.88 & 2.4 & 0.58 \\
\hline Focus/change & 0.76 & 0.28 & 1.35 & 0.38 & 1.27 & 0.41 & 3.30 & 0.98 & 3.7 & 0.65 \\
\hline Nonfocus/no change & 0.72 & 0.26 & 1.35 & 0.42 & 1.08 & 0.35 & 3.26 & 0.81 & 3.4 & 0.64 \\
\hline Nonfocus/change & 0.82 & 0.26 & 1.28 & 0.38 & 1.07 & 0.31 & 3.32 & 0.99 & 3.3 & 0.51 \\
\hline
\end{tabular}

Analysis of total time also showed a main effect of change $\left[F_{1}(1,31)=15.77, M S_{\mathrm{e}}=3,386, p<.001 ; F_{2}(1,23)=\right.$ 7.07, $\left.M S_{\mathrm{e}}=3,873, p<.05\right]$. Again, the pattern was for reading times to be longer when a change had taken place. There was a trend toward an interaction, which reached significance only in the items analysis $\left[F_{1}(1,31)=2.02\right.$, $M S_{\mathrm{e}}=9,772, p=.17 ; F_{2}(1,23)=4.26, M S_{\mathrm{e}}=4,491, p=$ $.05]$. This trend was due to the fact that total times were longer for changed than for nonchanged words when the word was in focus $\left[t_{1}(31)=2.80, p<.01 ; t_{2}(23)=3.04\right.$, $p<.01$ ], but not when the word was not in focus (both $t \mathrm{~s}<1)$. There were no other significant effects for the total time measure (all $F_{\mathrm{s}}<1.6$, all $p \mathrm{~s}>.2$ ).

Analysis of the number of fixations showed a main effect of change, with changed words receiving more fixations than unchanged words $\left[F_{1}(1,31)=6.36, M S_{\mathrm{e}}=\right.$ $\left.0.09, p<.05 ; F_{2}(1,23)=7.28, M S_{\mathrm{e}}=0.06, p<.05\right]$. However, this effect was modulated by focus, leading to a significant interaction between change and focus $\left[F_{1}(1,31)=6.02, M S_{\mathrm{e}}=0.11, p<.05 ; F_{2}(1,23)=4.57\right.$, $\left.M S_{\mathrm{e}}=0.09, p<.05\right]$. As with total times, the pattern was for the two focus conditions to differ, with more fixations in the focus/change than in the focus/no-change condition $\left[t_{1}(31)=3.371, p<.01 ; t_{2}(23)=2.97, p<.01\right]$, but the two nonfocus conditions did not differ (both $t \mathrm{~s}<1$ ). There was no main effect of focus in the number of fixations (both $F \mathrm{~s}<1.83$, both $p \mathrm{~s}>.18$ ).

We see then that the occurrence of a word change across the two presentations reliably affected reading times in measures of both early and later processing. The modulating effect of our focus manipulation was less robust, however, appearing only in the total number of fixations on a changed word and, marginally, in the total time measure. However, we believe that reading of the second display involved more parafoveal processing, because of both the highly predictable nature of previously read text and the longer saccade length involved in rereading (Raney \& Rayner, 1995). Thus, the critical word may have been processed parafoveally in a large number of trials in the second display. To examine this possibility, we ran an analysis of the probability of an initial fixation on the critical word.

The probability of initial fixation was calculated as the proportion of trials in which the critical word was fixated before being passed over for the first time. We computed this probability as being conditional on having made a prior fixation within 10 characters to the left of the critical word. This is because trials in which the previous fixation was far from the critical word are unlikely to reflect cases in which the word was processed parafoveally. Any effects of our manipulations on this measure would indicate that the critical word was processed prior to fixation. Table 3 shows the mean probability of fixation for the four conditions with the critical word.

There were no significant main effects of change or focus (all $F \mathrm{~s}<3.03$, all $p \mathrm{~s}>$.09). However, an interaction was observed that was significant by participants and marginal by items $\left[F_{1}(1,31)=4.69, M S_{\mathrm{e}}=1,938, p<\right.$ $\left..05 ; F_{2}(1,23)=3.46, M S_{\mathrm{e}}=1,226, p<.08\right]$. This interaction indicated that readers were more likely to make an initial fixation on the critical word when it both had changed and was in focus. There was a significant dif-

Table 3

Mean Probability of Initial Fixation on the Critical Word in Display 2

\begin{tabular}{|c|c|c|c|c|c|c|c|c|}
\hline & \multicolumn{2}{|c|}{$\begin{array}{c}\text { Focus/ } \\
\text { No Change }\end{array}$} & \multicolumn{2}{|c|}{$\begin{array}{l}\text { Focus/ } \\
\text { Change }\end{array}$} & \multicolumn{2}{|c|}{$\begin{array}{l}\text { Nonfocus/ } \\
\text { No Change }\end{array}$} & \multicolumn{2}{|c|}{$\begin{array}{c}\text { Nonfocus/ } \\
\text { Change }\end{array}$} \\
\hline & $M$ & $S D$ & $M$ & $S D$ & $M$ & $S D$ & $M$ & $S D$ \\
\hline Probability & .61 & .27 & .76 & .26 & .65 & .31 & .65 & .26 \\
\hline
\end{tabular}




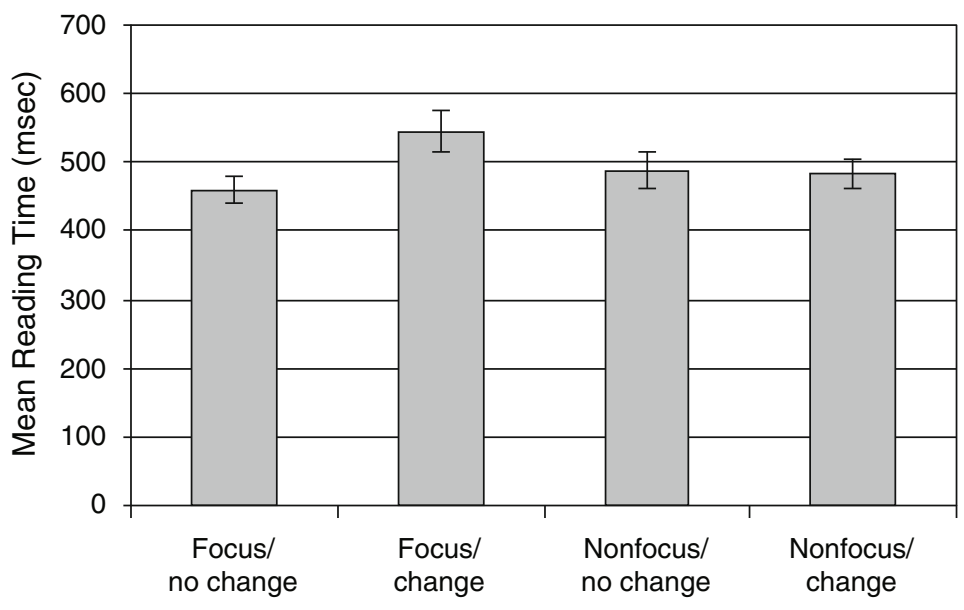

Figure 1. First-pass reading times (in milliseconds) for the extended region in the second display.

ference between the means for the focus/no-change and focus/change conditions in a comparison by participants $\left[t_{1}(31)=2.858, p<.01 ; t_{2}(23)=1.657, p>.05\right]$. There was no significant difference between the means for the nonfocus/no-change and nonfocus/change conditions (both $t \mathrm{~s}<1$ ).

Fixations on an extended critical region. The results for the probability of initial fixation show that the focus and change manipulations affected whether participants initially fixated the critical word or skipped over it. This shows that the critical word was being processed parafoveally, at least on some trials (Blanchard, Pollatsek, \& Rayner, 1989; Rayner, Well, Pollatsek, \& Bertera, 1982). Given that the critical word affected reading behavior prior to fixation, we decided to run the eye movement measures on an extended region consisting of the critical word plus the preceding region (Region 3 ). The new critical region would therefore be, for example,

(6) ... the seat [by the door] would give me a good view.

On the basis of this new region, we calculated first-pass times, total reading times, and number of fixations to provide measures of both initial and later processing that would take into account any information processed parafoveally. The means for the extended region are reported in Table 2.

First-pass analysis of the extended region yielded a main effect of change that was significant by participants only $\left[F_{1}(1,31)=5.50, M S_{\mathrm{e}}=9,497, p<.05 ; F_{2}(1,23)=2.16\right.$, $\left.M S_{\mathrm{e}}=13,900, p=.16\right]$. This effect was modulated by a significant interaction $\left[F_{1}(1,31)=6.22, M S_{\mathrm{e}}=10,309\right.$, $p=.05 ; F_{2}(1,23)=6.05, M S_{\mathrm{e}}=6,584, p<.05$; see Figure 1]. Again, reading times were particularly long when the critical word changed and was in focus. The difference in means between the focus/no-change and focus/change conditions was significant $\left[t_{1}(31)=2.965, p<.01\right.$; $\left.t_{2}(23)=2.400, p<.05\right]$, and the difference between the nonfocus/no-change and nonfocus/change conditions was not significant (both $t \mathrm{~s}<1$ ).
The results for total time showed a similar pattern. There was a main effect of change, which was significant in the participants analysis $\left[F_{1}(1,31)=8.49, M S_{\mathrm{e}}=7,811, p<\right.$ $\left..01 ; F_{2}(1,23)=3.45, M S_{\mathrm{e}}=12,599, p=.076\right]$. There was also a significant interaction between focus and change $\left[F_{1}(1,31)=4.56, M S_{\mathrm{e}}=14,997, p<.05 ; F_{2}(1,23)=\right.$ $\left.5.51, M S_{\mathrm{e}}=10,249, p<.05\right]$ : Readers spent longer reading the extended region in the focus/change condition than in the focus/no-change condition $\left[t_{1}(31)=3.073\right.$, $\left.p<.005 ; t_{2}(23)=2.995, p<.05\right]$, but the difference between the two nonfocus conditions was not reliable (both $t \mathrm{~s}<1$ ). There was no main effect of focus in the total time data (both $F \mathrm{~s}<3.2$, both $p \mathrm{~s}>.09$ ).

For total number of fixations, there was a main effect of focus in the analysis by items $\left[F_{1}(1,31)=2.56, M S_{\mathrm{e}}=\right.$ $\left.0.2, p=.12 ; F_{2}(1,23)=5.60, M S_{\mathrm{e}}=0.1, p<.05\right]$. The pattern was for more fixations to occur when the extended region was in focus. There was no main effect of change (both $F \mathrm{~s}<1.2)$ and a marginal interaction $\left[F_{1}(1,31)=\right.$ $4.03, M S_{\mathrm{e}}=0.2, p=.053 ; F_{2}(1,23)=3.35, M S_{\mathrm{e}}=0.2$, $p=.08]$. As in the data for the critical word alone, the pattern was for the region to receive more fixations in the change condition when it was in focus, but for the nonfocus conditions not to differ.

To summarize the fixation data for the first and second displays, we can say that the effect of focus in the first display was limited to regions of the sentence that preceded the critical word. However, in the second display, focus interacted with the effect of word change, with readers making both more fixations and fixations of a longer duration on the critical word, as well as on the extended critical region, when the critical word changed across displays and was in focus. This general finding is illustrated in Figure 2, which gives the data for total number of fixations on the critical word in both displays.

One interesting aspect of the results is that the nochange conditions did not differ as a function of focus in any of the analyses of the second display. One plausible hypothesis would be that focused items were more highly activated in memory than nonfocused items, lead- 


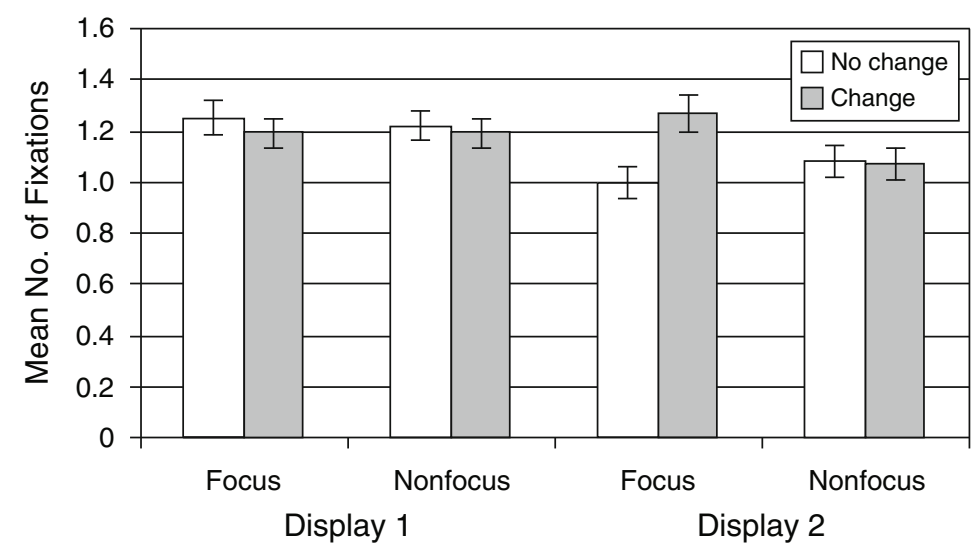

Figure 2. Total number of fixations on the critical word (both displays).

ing to a greater repetition advantage for the focused items across the two displays. This hypothesis predicts that focused items should be read more quickly than nonfocused items in the no-change conditions in the second display. However, this was not the case. In fact, our claim is that focus leads to an increased specificity of lexical meaning representation, and this does not necessarily imply increased activation in memory. Thus, our account does not predict an interaction between focus and repetition benefits. Note also that the lack of difference between the focus/no-change and nonfocus/no-change conditions in the second display implies the lack of a repetition effect between the first and second sentences in the second display, in contrast to our findings for the first display (recall that the head noun was repeated between the first and the second sentence in the focus condition, but not in the nonfocus condition, and that this led to a focus advantage in Region 2 in the first display). We do not have a straightforward explanation of this result. However, it is possible that such a repetition advantage may have reached ceiling level during the reading of the second display, since virtually every word was repeated from the first display, and this might have masked any local repetition advantage between the first and second sentences.

\section{Was Explicit Change Detection Affected by the Focus Manipulation?}

We wanted to know whether participants' explicit change detection performance was affected by focus. Recall that after reading both presentations of the text, the participants indicated their responses by pressing buttons corresponding to "change" and "no change." The granularity hypothesis discussed above predicts that change detection performance for the change conditions should

Table 4

Change Detection Performance (Percent Accuracy)

\begin{tabular}{|c|c|c|c|c|c|c|c|c|}
\hline & \multicolumn{2}{|c|}{$\begin{array}{c}\text { Focus/ } \\
\text { No Change }\end{array}$} & \multicolumn{2}{|c|}{$\begin{array}{l}\text { Focus/ } \\
\text { Change }\end{array}$} & \multicolumn{2}{|c|}{$\begin{array}{l}\text { Nonfocus/ } \\
\text { No Change }\end{array}$} & \multicolumn{2}{|c|}{$\begin{array}{l}\text { Nonfocus/ } \\
\text { Change }\end{array}$} \\
\hline & $M$ & $S D$ & $M$ & $S D$ & $M$ & $S D$ & $M$ & $S D$ \\
\hline Accuracy & 84.4 & 16.9 & 77.6 & 19.7 & 79.2 & 18.5 & 65.6 & 22.8 \\
\hline
\end{tabular}

be enhanced in the focus condition relative to the nonfocus condition, and this result would replicate Sturt et al.'s (2004) findings. However, no such focus effect is expected in the no-change conditions, because there is, by definition, no change in the semantics of the critical word, so representation at a greater or lesser degree of specificity should not be relevant. We calculated the percentage of correct answers for each condition (i.e., correct detections of actual changes and correct identifications of lack of a change). Mean accuracy rates for the four conditions are presented in Table 4.

ANOVAs indicated a main effect of focus, such that detection performance was better in the focus conditions $\left[F_{1}(1,31)=7.702, p<.05 ; F_{2}(1,23)=5.228, p<.05\right]$, and a main effect of change, such that performance was better for the no-change conditions $\left[F_{1}(1,31)=5.768\right.$, $\left.p<.05 ; F_{2}(1,23)=8.395, p<.01\right]$. Despite the lack of a reliable interaction (both $p \mathrm{~s}>.25$ ), the effect of focus appeared to have been driven entirely by the change conditions, with planned comparisons indicating higher detection rates for the focus/change than for the nonfocus/ change condition $\left[t_{1}(31)=2.777, p<.05 ; t_{2}(23)=\right.$ $2.064, p=.05]$. This difference replicates the effect of focus found by Sturt et al. (2004) for detection of close semantic changes. Accuracy for the two no-change conditions did not differ (both $t \mathrm{~s}<1.24$, both $p \mathrm{~s}>$.2). One remaining question is why overall accuracy was better for the no-change conditions. Given the task, possibly it was easier to detect the lack of a change than to detect an actual change. However, it should also be observed that the performance benefit for the no-change trials over the change trials was only substantial in the nonfocus condition (79.2\% vs. $65.6 \%$ for no-change vs. change trials), and this benefit was much reduced in the focus condition ( $84.4 \%$ vs. $77.6 \%$ for no-change vs. change). In fact, this conclusion is supported by $t$ tests: Accuracy was reliably greater for the no-change than for the change trials when the critical phrase was not in focus $\left[t_{1}(31)=2.57, p<\right.$ $\left..05 ; t_{2}(23)=2.78, p<.05\right]$. However, this difference was not reliable when the critical phrase was in focus (both $p s>.18)$. Thus, despite the lack of a reliable interaction, it may be that the apparent performance advantage for the 
no-change trials was driven entirely by the low performance in the nonfocus/change condition.

\section{Rereading/Repetition Effects}

Raney and Rayner (1995) found that readers made fewer and shorter fixations when reading a text for the second time. For the sake of completeness, we compared total reading times, total number of fixations, average fixation duration, and average forward saccade length across the whole passage for Displays 1 and 2, to see whether these repetition effects would be replicated in our study. An analysis of the total reading times indicated that readers took reliably less time overall to read the second display of the passage [6,467 vs. 4,932 msec for Displays 1 and 2, respectively; $F_{1}(1,31)=134.17, p<.001 ; F_{2}(1,23)=$ $1,401.65, p<.001]$. Similarly, analysis of the number of fixations indicated that readers made reliably fewer fixations when reading the second display [26.25 vs. 20.35 fixations; $F_{1}(1,31)=163.03, p<.001 ; F_{2}(1,23)=874.61$, $p<.001]$. Average fixation durations were longer for Display 1 than for Display 2 [249 vs. $244 \mathrm{msec} ; F_{1}(1,31)=$ $\left.6.74, p<.02 ; F_{2}(1,23)=20.20, p<.001\right]$, and finally, forward saccades were shorter for Display 1 than for Display 2 [9.7 vs. 10.9 characters; $F_{1}(1,31)=60.27, p<$ $\left..001 ; F_{2}(1,23)=288.85, p<.001\right]$.

\section{Implicit Effects of Change}

In the literature on change detection in natural scenes, there is some evidence for the implicit detection of changes. For example, Hollingworth and Henderson (2003) showed evidence for increased fixation times on changed objects even in trials in which the participant had failed to detect the change explicitly.

To test for any implicit effects of change in our linguistic stimuli, we analyzed trials in which participants failed to detect an actual change, and we compared these trials with those in which participants correctly identified that a change had not occurred. In other words, we concentrated the analysis on trials in which a participant made a "no change" response, and among these trials, we compared reading behavior between the trials in which a change did occur and those in which one did not. If Hollingworth and Henderson's (2003) findings generalize to linguistic stimuli, we should find longer fixation times for trials in which a change occurred than for those in which no change occurred.

Because change detection performance was high overall, the exclusion of correctly identified change trials led to the problem of sparse data. To solve this problem, we analyzed data only for the 19 participants from whom we had obtained eye movement data for a full set of four conditions in the extended region (i.e., Regions 3 and 4) after correctly identified change trials had been removed. Also because of the sparse-data problems, we will report $F_{1}$ analyses only (removal of a high proportion of experimental trials resulted in an unacceptable number of missing design cells in the items data; it was therefore not viable to compute an items $\left[F_{2}\right]$ analysis under these conditions). Table 5 gives the means for those participants once the relevant data were removed.
Table 5

Means for Undetected Change Trials and Correctly Detected No-Change Trials in the Extended Region $(N=19)$

\begin{tabular}{lll}
\hline & \multicolumn{2}{c}{$\begin{array}{c}\text { Extended Region } \\
\text { by the door }\end{array}$} \\
\cline { 2 - 3 } Measure & $M$ & $S D$ \\
\hline First Pass (msec) & & \\
Focus/no change & 444 & 126 \\
Focus/change & 623 & 389 \\
Nonfocus/no change & 477 & 171 \\
Nonfocus/change & 520 & 211 \\
Total Time (msec) & & \\
Focus/no change & 548 & 113 \\
Focus/change & 714 & 310 \\
Nonfocus/no change & 547 & 167 \\
Nonfocus/change & 677 & 344 \\
Number of Fixations & & \\
Focus/no change & 2.28 & 0.53 \\
Focus/change & 2.89 & 1.29 \\
Nonfocus/no change & 2.24 & 0.59 \\
Nonfocus/change & 2.64 & 1.12 \\
\hline
\end{tabular}

ANOVAs revealed that the combined region was fixated more often and for longer when a change was present than during trials in which no change was made [first pass, $F_{1}(1,18)=3.48, p<.08$; total time, $F_{1}(1,18)=13.01$, $p<.01$; number of fixations, $\left.F_{1}(1,18)=9.57, p<.01\right]$. However, neither the main effect of focus nor the focus $X$ change interaction was significant (all $p \mathrm{~s}>1$ ), indicating that detection of the changes at the implicit level was not significantly modulated by the focus manipulation. The failure to replicate the interaction could have resulted from the lower power associated with the reduced number of participants. Another possible explanation, however, relates to the link between focus and awareness: Perhaps focus affects only those words that comprehenders become aware of, and effects of purely automatic processing, such as implicit change detection, are dissociated from the typical benefits conferred by focus. At this point, the second idea can only be a conjecture, since the lower power of this analysis prevents us from making any strong conclusions.

The results for the missed-change trials therefore suggest that, as in visual processing, the language system can indeed register changes that do not reach conscious awareness, although the role of focus in such implicit detection of change remains unclear.

\section{GENERAL DISCUSSION}

Linguistic focus clearly had an influence both on reading behavior and change detection performance in our experiment. When a word change had occurred, participants were more accurate in reporting it when the changed word was in focus. In the second display of the text, readers' total number of fixations on the critical word was greatest when the word had changed and was in focus, relative to the other three conditions. This interaction was repeated for other measures, such as first-pass reading times, when the analysis region was extended to include fixations in which the critical word was processed parafoveally. We 
also observed an implicit effect of change in the eye movement record, indicating that participants could register word changes in the absence of conscious awareness. This effect is analogous to recent findings in visual cognition (see, e.g., Hollingworth \& Henderson, 2003).

The differences in processing difficulty that we observed across conditions in the second display (a slowdown when a focused word change occurred in the extended region, as well as a greater number of fixations on a focused word change) amount to a modulation of the repetition advantage observed by Raney and Rayner (1995; see above) for a second reading of text (see also Raney, Therriault, \& Minkoff, 2000). Although it is not currently known how differences in task (change detection vs. reading for comprehension) affect eye movement behavior, the effects of focus in our study point to differences in the semantic representations that readers built for focused and nonfocused target words. These differences can be interpreted as the result of focused target words having a finer-grained representation within the discourse representation, resulting in focused word changes being more easily detected and leading to longer and more numerous fixations. These findings are consistent with the literature on memory for focused items, and with Sturt et al.'s change detection study (2004; see above), in which the semantic distance of word changes was manipulated.

In terms of processing theories of focus, an interesting finding comes from the analysis of the first display of the text. Recall that the eye movement literature is at odds with itself over the question of how focus affects eye movements, with both Birch and Rayner (1997) and Morris and Folk (1998) reporting contradictory findings in measures of later processing, and Birch and Rayner reporting initial processing effects only when an entire phrase was in focus. We hoped that the analysis of eye fixations in first displays might bring some clarity on this issue. In fact, although we observed some faster reading of the regions preceding the critical word, which may have been partly due to repetition, no effects of focus on the critical word were found in the first display. Since effects of focus on the critical word were found in the second display, we can rule out an account in which the focus advantage is a direct result of longer or more numerous fixations in the first display, as has been suggested on the basis of Birch and Rayner's finding that participants spent a longer time processing focused information (see also Conrad \& Rips, 1986; Zimmer \& Engelkamp, 1981).

The data from our first display do not support the idea that focus leads to more effortful encoding in terms of longer or more numerous fixations. If anything, the results show that focus speeded up, rather than slowed down, processing in the first display, a result that is consistent with that of Morris and Folk (1998), although we observed this speedup only in the regions before our critical word, which could be explained in terms of repetition. The effect of focus on reading in the second display is consistent with the claim that focus leads to a more detailed semantic representation, since the processing system clearly reacts to the word change more strongly in focus than in nonfo- cus conditions. Taken together, these results for the first and second displays are consistent with a model in which focus leads to more specific encoding of meaning, yet this more specific encoding does not require greater effort.

One alternative to this possibility is that focus may indeed lead to more effortful encoding, but that the extra processing effort was masked in our experiment by a speedup associated with the repetition advantage in the focus conditions. Note, however, that we observed a speedup on the two regions preceding the critical word, but not on the critical word itself. Such a "masking" effect would thus have to have been localized on the critical word in order to explain our results. Furthermore, the idea that focus leads to more effortful encoding is not consistent with the results of Morris and Folk (1998), who found a speedup for focused phrases despite a lack of lexical repetition.

One question that remains is why the results of our study are different from those of Birch and Rayner (1997), who observed a slowdown in the reading of focused phrases. One possibility is that Birch and Rayner's effects could have been due to the different sentence frames in which the critical word appeared between the focus and nonfocus conditions.

For example, in Birch and Rayner's (1997) Experiment 1 , the critical word suburb appeared in the following sentence frames.

\section{(7) A. Focus}

It was the suburb that received the most damage from the ice storm.
B. Nonfocus
Workers in the suburb hurried to restore power after the ice storm.

Birch and Rayner reported longer second-pass reading times for the focus condition, which could potentially have been related to different probabilities of regression from the later part of the sentence between the two conditions. The present study used context focus, which held constant the sentence frame in which the target word appeared. This eliminated any potential confound between focus and the characteristics of the sentence frame.

Two other aspects of our study are worthy of note. First, we demonstrated that the focus manipulation had an effect on parafoveal processing behavior during the second reading of the text. Thus, focus can be added to the list of phenomena that affect processing when the relevant word is not directly fixated, although it remains to be seen whether focus could have a similar effect in the first reading of a text. A second interesting aspect of our study is the finding that word changes can affect processing behavior even in the absence of awareness, since total fixation times were longer for undetected changed words than for correctly detected unchanged words. This finding ties in with recent work on the implicit detection of change in the visual cognition literature (see Thornton \& Fernandez-Duque, 2002, for a review; see also Hollingworth \& Henderson, 2002, 2003; Hollingworth, Williams, \& Henderson, 2001).

We believe that we have demonstrated the effectiveness of a combination of the change detection task with eye 
movement recording for the investigation of processes relating to focus. Future work could exploit this technique to look more deeply into these issues by directly comparing different types of focusing devices.

We believe that the present study has marked several advances in our understanding of focus and eye movements in reading. Previous work cited above (Birch \& Rayner, 1997; Morris \& Folk, 1998) yielded conflicting results on the question of whether focus speeds up or slows down reading. In this article, we have concentrated on the question of how focus affects the specificity of representation, rather than on how focus affects reading speed per se. The results support the previous findings of Sturt et al. (2004), but that study did not involve eye movement recording, and so did not allow for inferences about the processing underlying change detection performance. The present study has contributed to answering this question, at least in relation to the manipulation of focus through context. The results show clearly that although focus does lead to more specific representations, this does not come about as the result of greater initial processing effort during the encoding stage. Also, our results show that the effect of focus on specificity is an online phenomenon, manifesting itself even in the parafoveal processing of focused elements, during the second reading of the display.

\section{AUTHOR NOTE}

This research was supported by ESRC Grant R000239888. We thank Tony Sanford for continual helpful discussions and comments on drafts of this article. We also thank the members of the language group at the Glasgow Psychology Department. This work was presented as a poster at the 2004 CUNY Conference on Human Sentence Processing in Maryland. We thank the audience for their comments and suggestions. Finally, we thank the reviewers for their helpful comments. Correspondence concerning this article should be addressed to P. Ward, Department of Psychology, University of Glasgow, Glasgow G12 8QQ, Scotland (e-mail: peter@psy.gla.ac.uk).

\section{REFERENCES}

Birch, S. L., \& Garnsey, S. M. (1995). The effect of focus on memory for words in sentences. Journal of Memory \& Language, 34, 232-267.

BIRCH, S. [L.], \& RAYNER, K. (1997). Linguistic focus affects eye movements during reading. Memory \& Cognition, 25, 653-660.

Blanchard, H. E., Pollatsek, A., \& Rayner, K. (1989). The acquisition of parafoveal word information in reading. Perception \& Psychophysics, 46, 85-94.

Bredart, S., \& Modolo, K. (1988). Moses strikes again: Focalization effect on a semantic illusion. Acta Psychologica, 67, 135-144.

British National CoRpus (1995). Available at www.natcorp.ox.ac.uk.

CHomsky, N. (1971). Deep structure, surface structure and semantic interpretation. In D. D. Steinberg \& L. A. Jakobovits (Eds.), Semantics: An interdisciplinary reader in philosophy, linguistics and psychology (pp. 183-216). Cambridge: Cambridge University Press.

Conrad, F. G., \& RIPS, L. J. (1986). Conceptual combination and the given/new distinction. Journal of Memory \& Language, 25, 255-278.

Cutler, A., \& Fodor, J. A. (1979). Semantic focus and sentence comprehension. Cognition, 7, 49-59.

Erickson, T. D., \& Mattson, M. E. (1981). From words to meaning:
A semantic illusion. Journal of Verbal Learning \& Verbal Behavior, 20, 540-551.

Ferreira, F., Bailey, K. G. D., \& Ferraro, V. (2002). Good-enough representations in language comprehension. Current Directions in Psychological Science, 11, 11-15.

Gergely, G. (1992). Focus-based inferences in sentence comprehension. In I. A. Sag \& A. Szabolcsi (Eds.), Lexical matters (pp. 47-65). Stanford: Stanford Center for the Study of Language \& Information.

Gernsbacher, M. A., \& Hargreaves, D. (1988). Accessing sentence participants: The advantage of first mention. Journal of Memory \& Language, 27, 699-717.

HallidaY, M. A. K. (1967). Notes on transitivity and theme in English, part 2. Journal of Linguistics, 3, 199-244.

Hobis, J. R. (1985, August). Granularity. Paper presented at the 9th International Joint Conference on Artificial Intelligence, Los Angeles.

Hollingworth, A., \& Henderson, J. M. (2002). Accurate visual memory for previously attended objects in natural scenes. Journal of Experimental Psychology: Human Perception \& Performance, 28, 113-136.

Hollingworth, A., \& Henderson, J. M. (2003). Testing a conceptual locus for the inconsistent object change detection advantage in realworld scenes. Memory \& Cognition, 31, 930-940.

Hollingworth, A., Williams, C. C., \& Henderson, J. M. (2001). To see and remember: Visually specific information is retained in memory from previously attended objects in natural scenes. Psychonomic Bulletin \& Review, 8, 761-768.

Morris, R. K., \& FolK, J. R. (1998). Focus as a contextual priming mechanism in reading. Memory \& Cognition, 26, 1313-1322.

Raney, G. E. (2003). A context-dependent representation model for explaining text repetition effects. Psychonomic Bulletin \& Review, 10, $15-28$.

RANEY, G. E., \& RAYNER, K. (1995). Word frequency effects and eye movements during two readings of a text. Canadian Journal of Experimental Psychology, 49, 151-172.

Raney, G. E., Therriault, D. J., \& Minkoff, S. R. B. (2000). Repetition effects from paraphrased text: Evidence for an integrated representation model of text representation. Discourse Processes, 29, 61-81.

Rayner, K., Well, A. D., Pollatsek, A., \& Bertera, J. H. (1982). The availability of useful information to the right of fixation in reading. Perception \& Psychophysics, 31, 537-550.

Rensink, R. A., O'Regan, J. K., \& Clark, J. J. (1997). To see or not to see: The need for attention to perceive changes in scenes. Psychological Science, 8, 368-373.

Rooth, M. (1995). Focus. In S. Lappin (Ed.), The handbook of contemporary semantic theory (pp. 271-298). Oxford: Blackwell.

SANFord, A. J., \& STURT, P. (2002). Depth of processing in language comprehension: Not noticing the evidence. Trends in Cognitive Sciences, 6, 382-386.

Sturt, P., Sanford, A. J., Stewart, A., \& Dawydiak, E. (2004). Linguistic focus and good-enough representations: An application of the change-detection paradigm. Psychonomic Bulletin \& Review, 11, 882-888.

Thornton, I. M., \& Fernandez-Duque, D. (2002). Converging evidence for the detection of change without awareness. In J. Hyönä, D. P. Munoz, W. Heide, \& R. Radach (Eds.), The brain's eye: Neurobiological and clinical aspects of oculomotor research (pp. 99-118). Amsterdam: Elsevier.

Zimmer, H. D., \& EngelKamp, J. (1981). The given-new structure of cleft sentences and their influence on picture viewing. Psychological Research, 43, 375-389.

\section{NOTE}

1. Post hoc analysis of the eye movement results excluding these items yielded data that were not substantially different from those reported below. 
APPENDIX

Experimental Materials

Across focus conditions, items differed only in the structure of the first sentence. In each item below, the first sentences for the focus and for the nonfocus condition are given in the first and the second bracketed sentence, respectively. In the second line of each item, the word change (occurring across displays in the change conditions) is represented, for instance, by a pair such as virus $\rightarrow$ infection, with virus appearing in the critical word position in the first display of the passage in the change conditions and infection appearing as the critical word in the second display. In the no-change conditions, the second word from each of these pairs was featured as the critical word in both the first and second displays of the passage.

Note-Double slash marks ( // ) indicate region boundaries. An asterisk before a number indicates that the word change features synonyms, according to the WordNet lexical database.

1.

\{The doctor checked to see which patient was next.\}\{The doctor checked to see how much longer he had to work.\}

He saw that the // patient // with the // virus $\rightarrow$ infection // was at the front of the queue. //

A kind but strict-looking nurse brought the boy in.

$* 2$.

$\{$ We all wondered which woman was the new employee. $\}\{$ We all wondered where the new employee was going.

It was obvious the // woman // carrying the // rucksack $\rightarrow$ backpack // was a bit lost. //

In such a big building it's so easy to lose your way.

3.

\{Tony heard which film had got the most awards at the ceremony.\}\{Tony heard all about the celebrities at the Oscar ceremony.\}

Apparently the // film // about the // aliens $\rightarrow$ martians // had been universally praised. //

Everybody thought it had been a wonderful ceremony.

4.

\{Simon really needed to decide which job to apply for.\}\{Simon really needed to decide what to do with his life.\}

He said that the // job // advertised in the // magazine $\rightarrow$ newspaper // had looked interesting. //

He really wanted something that would challenge him.

5.

\{The police still didn't know which boy had committed the crime.\}\{The police still didn't know how to proceed with investigations.

They thought the // boy // caught with the // lighter $\rightarrow$ matches // was a likely suspect. //

The witnesses had not been very helpful at all.

6.

\{We found out which tree the neighbours had removed.\}\{We found out what the neighbours had been up to.\}

The // tree // that had blocked the // street $\rightarrow$ road // had been cut down. //

It should make a real difference to their garden.

7.

\{The journalist wasn't sure which story he should write up. $\}\{$ The journalist wasn't sure what he should be doing.\}

He knew that the // story // about the // burglary $\rightarrow$ robbery // was long overdue. //

But his editor would be needing the front page picture.

8.

\{The lawyer wondered which document would be most helpful. $\}\{$ The lawyer wondered how he could construct a solid case.\}

Obviously the // document // for the // building $\rightarrow$ property // would be useful. //

He couldn't afford to let his client down.

9.

\{The taxi driver didn't know which house his customer lived in. \}\{The taxi driver didn't know where he was supposed to be.

Somehow the // house // with the // truck $\rightarrow$ lorry // in front seemed familiar. //

If he didn't find his way soon he would lose the customer. 


\section{APPENDIX (Continued)}

$* 10$.

$\{$ The secretary checked to see which letter had to be posted. $\}\{$ The secretary checked to see what had to be done next.\}

The // letter // to the // client $\rightarrow$ customer // was on the boss's desk. //

All the office chores had to be finished by five o' clock.

11.

\{The theatre critic was certain about which play would be popular.\}\{The theatre critic was certain about his latest recommendation.\}

He thought the // play // about the two // policemen $\rightarrow$ detectives // would run for months. //

He knew the theatre business and was usually right.

$* 12$.

\{The air traffic controller checked which plane was due for take-off.\}\{The air traffic controller checked that everything was running smoothly.

The // plane // carrying the important // packages $\rightarrow$ parcels // was approaching the runway. //

It could be quite a stressful job.

13.

$\{$ The advertising executive explained which poster would be successful. $\}\{$ The advertising executive explained how to reach the target audience.

He said the // poster // featuring the // kitten $\rightarrow$ puppy // was a safe bet. //

He had a lot of experience in the advertising industry.

14.

\{The ramblers thought they knew which path would take them home. $\}\{$ The ramblers thought they were getting near to the village.

It seemed that the // path // beside the // canal $\rightarrow$ stream // was going in the right direction. //

But without a detailed map there was no way to be certain.

15.

\{It became clear which reports had affected attitudes in the city. $\{$ It became clear how attitudes in the city had started to change. $\}$

The // reports // of the recent // killings $\rightarrow$ murders // had made the community more vigilant. //

But a heavy police presence would still be necessary.

16.

\{The fireman asked us which woman had raised the alarm.\}\{The fireman asked us how the incident had started.\}

We pointed out the // woman // wearing the // sweater $\rightarrow$ jumper // who had dialled 999. //

They wanted to get the full story.

17.

\{The crime squad guessed which building the criminal was hiding in. $\}$ The crime squad guessed the criminal was somewhere in the local area.

Soon the // building // behind the // pond $\rightarrow$ lake // was completely surrounded. //

But he was not found and the search continued for days.

18.

\{I couldn't decide which seat to take at the theatre. $\}$ I c couldn't decide whether I liked the new theatre layout. $\}$ I hoped the // seat // by the // exit $\rightarrow$ door // would give me a good view. //

It turned out to be a wonderful evening's entertainment.

$* 19$.

\{He asked me which ghost it was that I had actually seen.\}\{He asked me if I had ever had a supernatural experience.\}

I told him about the // ghost // in the // graveyard $\rightarrow$ cemetery // that had scared me. //

I don't think he believed me. 


\section{APPENDIX (Continued)}

20.

\{The vet wondered which dog was making all the noise.\} \{The vet wondered what all the noise was about.\}

The // dog // with the injured // legs $\rightarrow$ paws // would not stop barking. //

The owner was getting quite embarrassed.

21.

\{The student asked the professor which book she should read. $\}\{$ The student asked the professor for advice about the course. $\}$

He said that the // book // on ancient //rituals $\rightarrow$ ceremonies // would be essential. //

The student needed all the advice she could get.

22.

\{The museum owner wanted to know which box was missing from the exhibit.\}\{ The museum owner wanted to know about the preparations for the exhibit.

It turned out the // box // containing the old // drawing $\rightarrow$ painting // was still in the van. //

There would be terrible trouble if anything went missing.

23.

\{The student would have to choose which course to drop next year. $\}$ The student would have to choose very carefully this year. $\}$

The // course // containing // chemistry $\rightarrow$ biology // would probably have to be avoided. //

It was important to have a timetable with no clashes.

24.

\{The zookeeper knew which cage had to be cleaned next.\}\{The zookeeper knew he had some cleaning to do.\} He had noticed that the // cage // for the // tigers $\rightarrow$ lions // was beginning to smell. //

It was a big job and would probably take all day.

(Manuscript received December 12, 2004;

revision accepted for publication August 26, 2005.) 